\title{
Beyond the Thomas-Fermi approximation for a trapped condensed Bose-Einstein gas
}

\author{
Alexander L. Fetter \\ Departments of Physics and Applied Physics, Stanford University, Stanford, CA 94305-4060 U.S.A. \\ David L. Feder* \\ Department of Physics and Astronomy, McMaster University, Hamilton, Ontario L8S 4M1, Canada
}

(April 25, 2018)

\begin{abstract}
Corrections to the zero-temperature Thomas-Fermi description of a dilute interacting condensed Bose-Einstein gas confined in an isotropic harmonic trap arise due to the presence of a boundary layer near the condensate surface. Within the Bogoliubov approximation, the various contributions to the ground-state condensate energy all have terms of order $R^{-4} \ln R$ and $R^{-4}$, where $R$ is the number-dependent dimensionless condensate radius in units of the oscillator length $\sqrt{\hbar / m \omega_{0}}$. The zero-order hydrodynamic density-fluctuation amplitudes are extended beyond the Thomas-Fermi radius through the boundary layer to provide a uniform description throughout all space. The first-order correction to the excitation frequencies is shown to be of order $R^{-4}$.

03.75.Fi, 05.30.Jp, 32.80.Pj
\end{abstract}

\section{INTRODUCTION}

The recent experimental realization of Bose-Einstein condensation (BEC) of alkali-metal gases in magnetic traps [1 3] has generated great interest in the physics of a confined, interacting, dilute Bose gas. In the Bogoli-

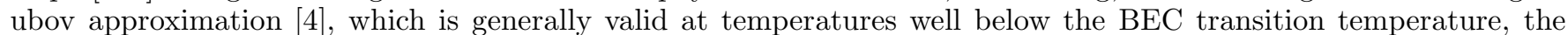
macroscopic occupation of the ground state far exceeds that of excited states; the condensate is then described by the relevant Gross-Pitaevskii (GP) equation [5]6]. This equation has been solved numerically for bosons in both isotropic [7 9] and anisotropic [10] traps, and analytically in the limits of both a nearly ideal gas (weak interparticle interactions) and a dilute nonideal gas (strong interparticle interactions) [11,12].

Given the resulting condensate wave function $\Psi$, the linearized small normal-mode amplitudes $u$ and $v$ satisfy the coupled Bogoliubov equations [6], which contain the condensate density $|\Psi|^{2}$ through the potential energy of interaction. An alternative hydrodynamic approach makes use of the fluctuations in the density and the velocity. It has been shown to be wholly equivalent to the Bogoliubov description 13 15, and it accurately describes the low-lying excited states [16 18], as recent experiments have verified [19,20] in considerable detail.

For large particle number, the mean kinetic energy of the condensate is much smaller than both the interaction (Hartree) and trap confinement energies. Neglecting the kinetic energy entirely, corresponding to the Thomas-Fermi (TF) approximation, provides an accurate description of the condensate in the interior of the cloud. Near the surface of the trapped gas, however, the kinetic and external potential energies become comparable, and the TF approximation breaks down. Using a boundary-layer theory, Dalfovo et al. 21] have calculated the kinetic energy as a function of the condensate radius; the formally divergent TF kinetic energy is cut off by a boundary layer of thickness $\delta \propto R^{-4 / 3}$, where $R$ is the (large) dimensionless TF condensate radius.

The present work extends the boundary-layer formalism of Ref. 21] to determine the leading-order corrections to the $\mathrm{TF}$ description of the condensate wave function, the condensate energy, and the low-lying collective modes. Section II summarizes the basic formalism and obtains the first correction to the condensate wave function, both in the bulk (of order $R^{-4}$ ) and in the boundary layer (of order $\delta$ ). In Sec. III, we show that this perturbative expansion gives rise to correction terms of relative order $R^{-4} \ln R$ and $R^{-4}$ in the normalization of the condensate wave function and in the trap and interaction energies of the condensate. A combination with the previously evaluated kinetic energy [21] provides the leading correction to the TF total condensate energy. In Sec. IV, we show that the structure of the condensate boundary layer plays an essential role in constructing the corresponding boundary layer for the analytical hydrodynamic normal modes [16]; the resulting hydrodynamic amplitudes for the density and current fluctuations

\footnotetext{
${ }^{*}$ Present address: Electron and Optical Physics Division, National Institute of Standards and Technology, Gaithersburg, MD 20899.
} 
vanish exponentially as $r \rightarrow \infty$, as expected from their equivalence to the eigenfunctions of the coupled Bogoliubov equations.

\section{CONDENSATE WAVE FUNCTION}

For a dilute interacting inhomogeneous Bose gas in an isotropic trap potential $V_{\text {ext }}$ at zero temperature, the total occupation of the excited states is small, and one can apply the Bogoliubov approximation [4,22]. The spatially varying condensate wave function $\Psi(\mathbf{r})$ is then isotropic and satisfies the (stationary) Gross-Pitaevskii [5] equation:

$$
\left(T+V_{\mathrm{ext}}+V_{\mathrm{H}}-\mu\right) \Psi(r)=0,
$$

where $T=-\hbar^{2} \nabla_{r}^{2} / 2 m$ is the kinetic energy, and the trap potential $V_{\text {ext }}(r)=m \omega_{0}^{2} r^{2} / 2$ is taken to be isotropic for simplicity. The Hartree energy is the mean energy of interaction of a particle at $\mathbf{r}$ with all the other particles, defined as $V_{\mathrm{H}}(r)=\int d^{3} r^{\prime} U\left(\mathbf{r}-\mathbf{r}^{\prime}\right)\left|\Psi\left(\mathbf{r}^{\prime}\right)\right|^{2} \approx g|\Psi(r)|^{2}$, where the last form reflects the short-range two-body interaction $U(\mathbf{r}) \approx g \delta^{(3)}(\mathbf{r})$. To leading order, the coupling constant $g=4 \pi a \hbar^{2} / m$ is written in terms of the (low-energy) $s$-wave scattering length $a$ to make contact with experiment; in the present work we only consider $a>0$, corresponding to interparticle repulsion. The chemical potential $\mu$ fixes the total number of condensed atoms $N_{0}=\int d^{3} r|\Psi(\mathbf{r})|^{2}$ through

$$
\mu N_{0}=\langle T\rangle+\left\langle V_{\text {ext }}\right\rangle+\left\langle V_{\mathrm{H}}\right\rangle
$$

where the noncondensate contribution to the chemical potential is neglected and $\langle\cdots\rangle \equiv \int d^{3} r \Psi^{*} \cdots \Psi$ denotes an expectation value in the condensate ground state.

It is convenient to use the the oscillator length $d_{0}=\sqrt{\hbar / m \omega_{0}}$ and the oscillator energy $\hbar \omega_{0}$ as the basic dimensional units. Thus we introduce the dimensionless length $z \equiv r / d_{0}$, and the dimensionless parameter

$$
\eta_{0}=\frac{N_{0} a}{d_{0}}
$$

that characterizes the strength of the interparticle interactions. Experimental conditions typically give $\eta_{0} \gg 1$, corresponding to a strongly interacting system [11] (but the Bogoliubov approximation still requires a dilute system with a large interparticle spacing $n^{-1 / 3}$ relative to $a$ ). In this TF limit, the repulsive interactions expand the condensate cloud beyond $d_{0}$ to a large dimensionless radius $R \propto N_{0}^{1 / 5}$ (expressed in units of $d_{0}$ ) [11]. The kinetic energy in Eq. (1) then becomes small, and the condensate density has a simple parabolic form $n_{0}=|\Psi|^{2} \approx g^{-1}\left(\mu-V_{\text {ext }}\right)=$ $(\mu / g)\left(1-z^{2} / R^{2}\right)$. Since $\eta_{0} \propto N_{0}$ scales like $R^{5}$, it is convenient to define

$$
\eta_{0} \equiv \tilde{\eta}_{0} R^{5}
$$

where $\tilde{\eta}_{0}$ approaches a constant value for large $R$. Defining a scaled variable $x=z / R$, the full Gross-Pitaevskii equation (11) becomes:

$$
\left[-\frac{1}{2} \epsilon \nabla_{x}^{2}+\frac{1}{2} x^{2}+|\tilde{\Psi}(x)|^{2}-\tilde{\mu}\right] \tilde{\Psi}(x)=0,
$$

where $\epsilon=1 / R^{4}$ is a small coefficient in the present limit $R \rightarrow \infty$ and $\tilde{\mu}=\mu / R^{2}$ is the scaled chemical potential. Here, the scaled condensate wave function

$$
|\tilde{\Psi}|^{2} \equiv \frac{4 \pi d_{0}^{3} \eta_{0}}{N_{0} R^{2}}|\Psi|^{2}=\frac{4 \pi d_{0}^{3} R^{3} \tilde{\eta}_{0}}{N_{0}}|\Psi|^{2}
$$

becomes independent of $R$ for large $R$.

The normalization of the condensate wave function is then written

$$
\tilde{\eta}_{0} \equiv \frac{\eta_{0}}{R^{5}}=\int_{0}^{\infty} d x x^{2}|\tilde{\Psi}(x)|^{2}
$$

which defines the condensate radius in terms of the particle number. Correspondingly, the scaled chemical potential follows ifrom Eq. (2) as $\tilde{\mu}=\left\langle\tilde{V}_{\text {ext }}+\tilde{V}_{H}+\tilde{T}\right\rangle$, where

$$
\left\langle\tilde{V}_{\text {ext }}\right\rangle \equiv \frac{\left\langle V_{\text {ext }}\right\rangle}{R^{2}}=\frac{N_{0}}{2 \tilde{\eta}_{0}} \int_{0}^{\infty} d x x^{4}|\tilde{\Psi}(x)|^{2}
$$




$$
\begin{aligned}
& \left\langle\tilde{V}_{\mathrm{H}}\right\rangle \equiv \frac{\left\langle V_{\mathrm{H}}\right\rangle}{R^{2}}=\frac{N_{0}}{\tilde{\eta}_{0}} \int_{0}^{\infty} d x x^{2}|\tilde{\Psi}(x)|^{4} ; \\
& \langle\tilde{T}\rangle \equiv \frac{\langle T\rangle}{R^{2}}=\frac{N_{0} \epsilon}{2 \tilde{\eta}_{0}} \int_{0}^{\infty} d x x^{2}\left|\tilde{\Psi}^{\prime}(x)\right|^{2},
\end{aligned}
$$

where all the energies are expressed in units of $\hbar \omega_{0}$, and $\tilde{\Psi}^{\prime}=d \tilde{\Psi} / d x$ is the scaled radial derivative.

In the Thomas-Fermi (TF) limit, one sets the small parameter $\epsilon$ to zero in Eq. (5). The approximate condensate wave function $\tilde{\Psi}(x) \approx \tilde{\Psi}_{\mathrm{TF}}(x) \theta(1-x)=\sqrt{\frac{1}{2}\left(1-x^{2}\right)} \theta(1-x)$ accurately describes the condensate in the bulk [10,11, where we take $\tilde{\mu}=\frac{1}{2}$, defining the condensate radius by $R(\mu)=\sqrt{2 \mu}$. With this choice, the normalization condition Eq. (7) then determines the condensate number $N_{0}$ in terms of the radius $R$ and the chemical potential.

The Thomas-Fermi approximation fails near the surface at $x=1$, however, where $\tilde{V}_{\text {ext }}=\frac{1}{2} x^{2}$ is comparable to $\tilde{\mu}$; in this region, the kinetic term in Eq. (5) becomes significant, giving rise to a logarithmic divergence in Eq. (10). For small positive $\epsilon$, a boundary layer of thickness $\delta$ forms in the vicinity of $x=1$ where the condensate wave function varies rapidly. Using standard techniques in boundary-layer theory [23], we define an outer solution $\tilde{\Psi}_{\text {outer }} \equiv \chi(x)$ valid in the bulk region $0 \leq x \leq x_{0}<1$, and an inner solution $\tilde{\Psi}_{\text {inner }}$, valid throughout the surface region $x_{0} \leq x \leq \infty$; an asymptotic analysis matches these two solutions near the boundary $x \approx x_{0}$.

The outer (bulk) solution $\chi$ may be expressed as a perturbation series in powers of $\epsilon$ :

$$
\chi(x)=\chi_{0}(x)+\epsilon \chi_{1}(x)+\cdots, \quad 0 \leq x \leq x_{0} .
$$

Substituting Eq. (11) into Eq. (5) yields

$$
\begin{array}{ll}
O\left(\epsilon^{0}\right): & \chi_{0}^{2}(x)=\frac{1}{2}\left(1-x^{2}\right) \\
O\left(\epsilon^{1}\right): & 2 \chi_{0}(x) \chi_{1}(x)=\frac{\nabla_{x}^{2} \chi_{0}}{2 \chi_{0}}=\frac{x^{2}-\frac{3}{2}}{\left(x^{2}-1\right)^{2}},
\end{array}
$$

where we have set $\tilde{\mu}=\frac{1}{2}$ and specialized to the present case of a real isotropic condensate wave function. In order to determine the asymptotic behavior of the outer solution near the boundary, one may write $x=1+\delta X$ with $\delta|X| \ll 1$ and $|X| \gg 1$ (where $X$ is large and negative). As $X \rightarrow-\infty$, a straightforward calculation reveals

$$
\chi(X) \sim \delta^{1 / 2}(-X)^{1 / 2}\left(1+\frac{1}{8 X^{3}}\right)-\frac{\delta^{3 / 2}(-X)^{3 / 2}}{4}\left(1-\frac{21}{8 X^{3}}\right)+\cdots,
$$

where the leading-order behavior $\left(\propto \delta^{1 / 2}\right)$ agrees with previous calculations [24].

The asymptotic behavior of the outer solution implies that the inner solution has the form $\tilde{\Psi}_{\text {inner }}(X) \equiv \delta^{1 / 2} \Phi(X)$; it may be expanded as a series in $\delta$ :

$$
\tilde{\Psi}_{\text {inner }}(X)=\delta^{1 / 2}\left[\Phi_{0}(X)+\delta \Phi_{1}(X)+\cdots\right]
$$

where $X_{0} \leq X<\infty$ and $X_{0}$ is large and negative. Substituting Eq. (15) into Eq. (5) immediately gives the "distinguished limit" [23] $\epsilon=2 \delta^{3}$ that balances the leading-order (gradient and nonlinear) terms in the resultant differential equation. We readily obtain:

$$
\begin{array}{ll}
O\left(\delta^{0}\right): & \Phi_{0}^{\prime \prime}(X)-\left[X+\Phi_{0}^{2}(X)\right] \Phi_{0}(X)=0 \\
O\left(\delta^{1}\right): & \Phi_{1}^{\prime \prime}(X)-\left[X+3 \Phi_{0}^{2}(X)\right] \Phi_{1}(X)=-2 \Phi_{0}^{\prime}(X)+\frac{1}{2} X^{2} \Phi_{0} .
\end{array}
$$

It is straightforward to verify that, for $X \rightarrow-\infty$, the inner functions $\Phi(X)$ exactly reproduce Eq. (14):

$$
\begin{aligned}
& \Phi_{0}(X) \sim(-X)^{1 / 2}\left(1+\frac{1}{8 X^{3}}\right) ; \\
& \Phi_{1}(X) \sim-\frac{(-X)^{3 / 2}}{4}\left(1-\frac{21}{8 X^{3}}\right) .
\end{aligned}
$$

For large positive $X, \tilde{\Psi}_{\text {inner }}(X)$ is asymptotically proportional to an Airy function and therefore decays exponentially. Indeed, since Eq. (16) defines what is known as the second Painlevé transcendent, the boundary condition $\Phi_{0}(X \rightarrow$ $\infty) \sim \sqrt{2} \operatorname{Ai}(X) \sim(2 \pi)^{-1 / 2} X^{-1 / 4} \exp \left(-\frac{2}{3} X^{3 / 2}\right)$ must be imposed in order to ensure an unbounded solution for $\Phi_{0}(X)$ with no critical points over all $X$ [25]. 


\section{CONDENSATE ENERGIES}

The normalization of the condensate wave function can now be determined explicitly from Eq. (7) by separating the integral into two parts at $x_{0}$. An expansion of each contribution shows that the resulting sum is independent of the matching point $x_{0}=1+\delta X_{0}$ and yields

$$
\tilde{\eta}_{0} \approx \frac{1}{15}+\delta^{2} I+\frac{1}{8} \epsilon \ln \epsilon+\epsilon\left(\frac{1}{24}-\frac{\ln 2}{2}+J\right)
$$

where $I$ and $J$ are definite integrals involving the inner function $\Phi_{0}(X)$ (see Appendix A for details). In fact, $I$ can be shown to vanish identically, so that the leading corrections are of order $R^{-4} \ln R$ and $R^{-4}$ (instead of order $R^{-8 / 3}$ implicit in the term of order $\delta^{2}$ ).

A similar analysis yields the physical quantities in Eqs. (8)-(10):

$$
\begin{gathered}
\left\langle\tilde{V}_{\text {ext }}\right\rangle \approx \frac{N_{0}}{2 \tilde{\eta}_{0}}\left[\frac{1}{35}+\delta^{2} I+\frac{1}{24} \epsilon \ln \epsilon+\epsilon\left(\frac{5}{24}-\frac{\ln 2}{6}+K\right)\right], \\
\left\langle\tilde{V}_{\mathrm{H}}\right\rangle \approx \frac{N_{0}}{\tilde{\eta}_{0}}\left[\frac{2}{105}+\frac{1}{12} \epsilon \ln \epsilon+\epsilon\left(-\frac{\ln 2}{3}+\frac{L}{2}\right)\right], \\
\langle\tilde{T}\rangle \approx-\frac{N_{0}}{\tilde{\eta}_{0}}\left[\frac{1}{24} \epsilon \ln \epsilon+\epsilon\left(\frac{1}{12}-\frac{\ln 2}{6}+\frac{M}{2}\right)\right],
\end{gathered}
$$

where the constants $K, L$, and $M$ are also definite integrals involving the inner function $\Phi_{0}(X)$. A combination of analytical and numerical techniques gives the explicit results

$$
\begin{aligned}
I & =0 ; \\
J & =\frac{3}{4} L-\frac{5}{24} ; \\
K & =\frac{1}{4} L-\frac{7}{24} ; \\
L & \approx 0.4539 ; \\
M & =\frac{1}{2} L-\frac{1}{12},
\end{aligned}
$$

such that $K+L=J+M$.

Inserting the explicit expressions from Eqs. (21)-(23) into Eq. (2), we immediately recover $\mu=\frac{1}{2} R^{2}$, demonstrating the internal consistency of the calculation. Equation (20) implies

$$
\eta_{0}(R)=\left(\frac{a}{d_{0}}\right) N_{0} \approx \frac{R^{5}}{15}-\frac{R}{2}\left[\ln \left(\frac{R}{A}\right)+\frac{7}{12}\right] \approx \frac{R^{5}}{15}-\frac{R}{2} \ln (1.4128 R),
$$

where the constant $A$ is

$$
A=\frac{1}{2} \exp \left(\frac{3}{2} L+\frac{1}{4}\right) \approx 1.268
$$

This equation (29) relates the condensate number to the chemical potential (and hence the condensate radius); conversely, its inverse

$$
R\left(\eta_{0}\right) \approx\left(15 \eta_{0}\right)^{1 / 5}+\frac{3}{10}\left(15 \eta_{0}\right)^{-3 / 5} \ln \left(84.46 \eta_{0}\right)
$$

relates the radius of the cloud (and the chemical potential) to the condensate number. In each case, the first terms correspond to the TF result [11].

The various contributions to the total energy [Eqs. (8)-(10)] yield

$$
\begin{aligned}
& \frac{\left\langle V_{\mathrm{ext}}\right\rangle}{N_{0}} \approx \frac{3 R^{2}}{14}\left[1+\frac{5}{3 R^{4}} \ln \left(\frac{R}{A}\right)\right] ; \\
& \frac{\left\langle V_{\mathrm{H}}\right\rangle}{N_{0}} \approx \frac{2 R^{2}}{7}\left[1-\frac{10}{R^{4}} \ln \left(\frac{R}{A}\right)\right] ;
\end{aligned}
$$




$$
\frac{\langle T\rangle}{N_{0}} \approx \frac{5}{2 R^{2}} \ln \left(\frac{R}{A}\right)
$$

and the energies are expressed in units of $\hbar \omega_{0}$.

The expression for the kinetic energy in Eq. (34) is a small correction that involves only the leading contributions to the bulk condensate wave function and the boundary layer; it reproduces the result of Dalfovo et al. [21], and is similar to that found in 24. It should be kept in mind, however, that the present calculation defines the condensate radius $R$ in terms of the chemical potential $\mu \equiv \frac{1}{2} R^{2}$; it therefore includes correction terms beyond the TF approximation, as shown in Eq. (31).

The remaining contributions Eqs. (32) and (33) explicitly require the leading corrections $\chi_{1}$ and $\Phi_{1}$ and have not been evaluated previously. The dimensionless total energy per particle $\langle E\rangle / N_{0}=\left\langle T+V_{\text {ext }}+\frac{1}{2} V_{\mathrm{H}}\right\rangle$ is found to be

$$
\frac{\langle E\rangle}{N_{0}} \approx \frac{5 R^{2}}{14}\left[1+\frac{4}{R^{4}} \ln \left(\frac{R}{A}\right)\right]
$$

where the first and second terms are, respectively, the standard TF result and the combined contribution from both the boundary layer and the first correction to the bulk condensate wave function. Equations (29) and (35) together constitute a parametric representation of the relation $\left\langle E\left(N_{0}\right)\right\rangle[26]$, and it is not difficult to verify that $\mu=d\langle E\rangle / d N_{0}$.

In fact, Stringari 16,27 has noted that Eqs. (32)-(33) and (35) can be obtained solely from the expression for the kinetic energy (34). The virial theorem implies that the three contributions to the total energy must satisfy the condition

$$
2\langle T\rangle+\frac{3}{2}\left\langle V_{\mathrm{H}}\right\rangle-2\left\langle V_{\mathrm{ext}}\right\rangle=0
$$

The leading-order contributions to the potential energies $\left\langle V_{\text {ext }}\right\rangle$ and $\left\langle V_{\mathrm{H}}\right\rangle$ are known from TF theory, and the correction terms must have the same $R$-dependence as the kinetic energy but with unknown coefficients. Equation (36) and the condition $\mu=\partial\langle E\rangle / \partial N_{0}$ together determine for the unknown coefficients, reproducing the expressions found above by direct integration.

As shown in Fig. 1, the results of the boundary-layer theory agree strikingly with exact results obtained by integrating the GP equation (5) numerically. The analytical slow $R^{-2} \ln R$ decay of the average kinetic energy per particle (shown dashed bold) with the universal scaling parameter $\eta_{0}=N_{0} a / d_{0}$ accurately captures the behavior obtained numerically (shown solid bold). As a result, the boundary-layer theory provides a much better estimate of the total energy per particle (and therefore the chemical potential) than does the TF approximation. In spite of the slow decrease in the magnitude of $\langle T\rangle / N_{0}$ with the number of particles, however, it should be emphasized that the $\mathrm{TF}$ approximation to the total energy and the chemical potential is correct to better than $1 \%$ when $\eta_{0} \sim 1000$, due to their $R^{2}$ increase.

\section{EXCITED-STATE WAVE FUNCTIONS}

In the Bogoliubov approximation at zero temperature, the interparticle repulsions excite only a small fraction of all the particles out of the ground state into self-consistent normal modes. The resulting eigenfunctions $u_{j}(\mathbf{x})$ and $v_{j}(\mathbf{x})$ and eigenvalues $E_{j}$ for the noncondensate modes satisfy the coupled linear Bogoliubov equations [4, 6, 22]. For the present purpose, it is convenient to factor out the exact (real) GP condensate wave function $\Psi$, defining sum and difference amplitudes [15]

$$
F_{j}(\mathbf{x}) \equiv \frac{u_{j}(\mathbf{x})+v_{j}(\mathbf{x})}{\Psi(\mathbf{x})} \quad \text { and } \quad G_{j}(\mathbf{x}) \equiv \frac{u_{j}(\mathbf{x})-v_{j}(\mathbf{x})}{\Psi(\mathbf{x})}
$$

which are essentially the hydrodynamic amplitudes. Specifically, the perturbation in the velocity potential $\phi_{j}$ is proportional to $F_{j}$ (so that the perturbation in the current density is proportional to $\Psi^{2} \nabla F_{j}$ ) and the density perturbation $\rho_{j}$ is proportional to $\Psi^{2} G_{j}$. In the TF limit, it is convenient to rescale these amplitudes, with $F_{j}=R \tilde{F}_{j}$ and $G_{j}=\tilde{G}_{j} / R$, yielding the coupled linear equations

$$
\begin{aligned}
-\frac{1}{2} \boldsymbol{\nabla} \cdot\left(\tilde{\Psi}^{2} \nabla \tilde{F}_{j}\right) & =E_{j} \tilde{\Psi}^{2} \tilde{G}_{j}, \\
2 \tilde{\Psi}^{4} \tilde{G}_{j}-\frac{1}{2} \epsilon \nabla \cdot\left(\tilde{\Psi}^{2} \nabla \tilde{G}_{j}\right) & =E_{j} \tilde{\Psi}^{2} \tilde{F}_{j},
\end{aligned}
$$

where $\tilde{\Psi}^{2}$ is the (real) scaled condensate density from Eq. (6). These Eqs. (38) and (39) are self-adjoint with a normalization integral $\int d^{3} x \tilde{\Psi}^{2}\left(\tilde{F}_{j}^{*} \tilde{G}_{j}+\tilde{G}_{j}^{*} \tilde{F}_{j}\right)=1$ chosen to ensure positive energies $E_{j}$ for the stable solutions [22]. 
In the TF regime $(R \rightarrow \infty)$, Stringari [16] has solved these coupled equations for a stationary isotropic condensate, but the resulting hydrodynamic eigenfunctions are defined only inside the condensate $(x \leq 1)$. In contrast, the original Bogoliubov equations are well-defined throughout the whole region, including the classically forbidden region $(x \gg 1)$. Thus it is interesting to develop a boundary-layer description of the excited states similar to that for the condensate.

\section{A. Outer (bulk) region}

In the "outer" region $\left(0 \leq x \leq x_{0}<1\right)$, we follow the procedure for the condensate and expand the outer scaled amplitudes (37) and eigenvalues in powers of $\epsilon$ :

$$
\begin{aligned}
\tilde{F}_{j} & \approx \tilde{F}_{j}^{0}+\epsilon \tilde{F}_{j}^{\epsilon}+\ldots ; \\
\tilde{G}_{j} & \approx \tilde{G}_{j}^{0}+\epsilon \tilde{G}_{j}^{\epsilon}+\ldots ; \\
E_{j} & \approx E_{j}^{0}+\epsilon E_{j}^{\epsilon}+\ldots
\end{aligned}
$$

Substituting these expressions into Eqs. (38) and (39), and including the outer expansion of the condensate given in Eq. (11), one obtains:

$$
\begin{array}{ll}
O\left(\epsilon^{0}\right): \quad-\nabla \cdot\left(\chi_{0}^{2} \nabla \tilde{F}_{j}^{0}\right)-\left(E_{j}^{0}\right)^{2} \tilde{F}_{j}^{0}=0 \quad ; \quad \tilde{G}_{j}^{0}=\frac{E_{j}^{0} \tilde{F}_{j}^{0}}{2 \chi_{0}^{2}} ; \\
O\left(\epsilon^{1}\right): \quad-\nabla \cdot\left(\chi_{0}^{2} \nabla \tilde{F}_{j}^{\epsilon}\right)-\left(E_{j}^{0}\right)^{2} \tilde{F}_{j}^{\epsilon}=\frac{\left(E_{j}^{0}\right)^{2}}{4 \chi_{0}^{2}} \nabla \cdot\left[\chi_{0}^{2} \nabla\left(\frac{\tilde{F}_{j}^{0}}{\chi_{0}^{2}}\right)\right]+2 \nabla \cdot\left(\chi_{0} \chi_{1} \nabla \tilde{F}_{j}^{0}\right)+2 E_{j}^{0} E_{j}^{\epsilon} \tilde{F}_{j}^{0} ; \\
\tilde{G}_{j}^{\epsilon}=\frac{E_{j}^{0}}{8 \chi_{0}^{4}} \nabla \cdot\left[\chi_{0}^{2} \nabla\left(\frac{\tilde{F}_{j}^{0}}{\chi_{0}^{2}}\right)\right]+\frac{1}{2 \chi_{0}^{2}}\left(E_{j}^{0} \tilde{F}_{j}^{\epsilon}+E_{j}^{\epsilon} \tilde{F}_{j}^{0}\right)-\frac{E_{j}^{0} \chi_{1} \tilde{F}_{j}^{0}}{\chi_{0}^{3}} .
\end{array}
$$

The spherical symmetry of the condensate density permits a decomposition into spherical harmonics, with the normal-mode amplitudes proportional to $Y_{l m}(\theta, \phi)$. Equations (43) may then be solved explicitly, and the corresponding (unnormalized) zeroth-order radial amplitudes can be taken as

$$
\tilde{F}_{n l}^{0}(x) \equiv \rho_{n l}^{0}(x)=x^{l} P_{n l}\left(x^{2}\right) \quad ; \quad \tilde{G}_{n l}^{0}=\frac{E_{n l}^{0} \tilde{F}_{n l}^{0}}{2 \chi_{0}^{2}},
$$

where the radial quantum number $n$ denotes the number of nodes. Here the eigenvalues take the well-known result [16]

$$
E_{n l}^{0}=\sqrt{l+n(2 n+2 l+3)},
$$

and $P_{n l}\left(x^{2}\right)=F\left(-n, n+l+\frac{3}{2} ; l+\frac{3}{2} ; x^{2}\right)$ is a hypergeometric function [28] that terminates to give a polynomial of order $x^{2 n}$.

While analytical solutions to the inhomogeneous differential equations (44) and (45) are not easy to find, the asymptotic behavior of all the outer solutions in the vicinity of the boundary layer $x \sim 1$ may be readily ascertained. Setting $x=1+\delta X$ with $X \ll-1$ and expanding through $O\left(\delta^{3}\right)$, we obtain:

$$
\begin{aligned}
& O\left(\epsilon^{0}\right): \quad \tilde{F}_{n l}^{0} \sim \rho_{n l}^{0}(1)+\delta X \rho_{n l}^{0}{ }^{\prime}(1)+\frac{1}{2} \delta^{2} X^{2} \rho_{n l}^{0}{ }^{\prime \prime}(1)+\frac{1}{6} \delta^{3} X^{3} \rho_{n l}^{0}{ }^{\prime \prime \prime}(1) ; \\
& \tilde{G}_{n l}^{0} \sim-\frac{E_{n l}^{0}}{2 \delta X}\left\{G_{a}^{0}+\delta X G_{b}^{0}+\delta^{2} X^{2} G_{c}^{0}+\delta^{3} X^{3} G_{d}^{0}\right\} ; \\
& O\left(\epsilon^{1}\right): \quad \tilde{F}_{n l}^{\epsilon} \sim \frac{F_{a}^{\epsilon}}{\delta^{3} X^{3}}+\frac{F_{b}^{\epsilon}}{\delta^{2} X^{2}}+\frac{F_{c}^{\epsilon}}{\delta X}+F_{d}^{\epsilon}+F_{e}^{\epsilon} \ln (-\delta X)+F_{f}^{\epsilon} \ln ^{2}(-\delta X) ; \\
& \tilde{G}_{n l}^{\epsilon} \sim-\frac{E_{n l}^{0}}{2 \delta X}\left\{\frac{G_{a}^{\epsilon}}{\delta^{3} X^{3}}+\frac{G_{b}^{\epsilon}}{\delta^{2} X^{2}}+\frac{G_{c}^{\epsilon}}{\delta X}+G_{d}^{\epsilon}+G_{e}^{\epsilon} \ln (-\delta X)+G_{f}^{\epsilon} \ln ^{2}(-\delta X)\right\}-\frac{E_{n l}^{\epsilon} \rho_{n l}^{0}(1)}{2 \delta X},
\end{aligned}
$$

where the constants $F_{a-f}^{\epsilon}$ and $G_{a-f}^{0, \epsilon}$ are relegated to Appendix B for clarity. The expansion (48) is the conventional Taylor series of the zeroth-order amplitudes $\tilde{F}_{n l}^{0}(x)$ about $x=1$, where a prime denotes a derivative evaluated at $x=1$. A straightforward calculation following from the properties of the hypergeometric function [15,28] shows that 


$$
\rho_{n l}^{0}(1)=(-1)^{n} \frac{\Gamma\left(l+\frac{3}{2}\right) n !}{\Gamma\left(n+l+\frac{3}{2}\right)} \quad ; \quad \rho_{n l}^{0{ }^{\prime}}(1)=\left(E_{n l}^{0}\right)^{2} \rho_{n l}^{0}(1) \quad \ldots
$$

with higher-order values listed in Appendix B. Since $\epsilon=2 \delta^{3}$, the outer solutions in the region $x \sim 1$ are respectively $\tilde{F}_{n l} \approx \tilde{F}_{n l}^{0}+2 \delta^{3} \tilde{F}_{n l}^{\epsilon}$ and $\tilde{G}_{n l} \approx \tilde{G}_{n l}^{0}+2 \delta^{3} \tilde{G}_{n l}^{\epsilon}$.

\section{B. Inner (boundary-layer) region}

The need for a boundary-layer description of the eigenfunctions is clear from the form of condensate density $\tilde{\Psi}(x)^{2}=\delta \Phi(X)^{2}$ and the gradient $\boldsymbol{\nabla}_{x}=\delta^{-1} \boldsymbol{\nabla}_{X}$ (arising from the substitution $x=1+\delta X$ ). As a result, the second term on the left-hand side of Eq. (39) is now of order $\epsilon \times \delta / \delta^{2} \sim \delta^{2}$, which is wholly comparable with the first term and thus no longer negligible in zero order. It is not difficult to see that the proper scaling of the two inner amplitudes in the boundary layer is

$$
\tilde{F}_{n l}(x)=A_{n l}(X) \quad \text { and } \quad \tilde{G}_{n l}(x)=\frac{B_{n l}(X)}{\delta} .
$$

The gradients in Eqs. (38) and (39) must be expanded in ascending powers of $\delta$, leading to the following coupled equations for the inner solutions

$$
\begin{gathered}
-\frac{d}{d X}\left(\Phi^{2} \frac{d A_{n l}}{d X}\right)-\delta \frac{2 \Phi^{2}}{1+\delta X} \frac{d A_{n l}}{d X}+\delta^{2} \frac{l(l+1) \Phi^{2}}{(1+\delta X)^{2}} A_{n l}=2 \delta E_{n l} \Phi^{2} B_{n l} \\
-\frac{d}{d X}\left(\Phi^{2} \frac{d B_{n l}}{d X}\right)-\delta \frac{2 \Phi^{2}}{1+\delta X} \frac{d B_{n l}}{d X}+\delta^{2} \frac{l(l+1) \Phi^{2}}{(1+\delta X)^{2}} B_{n l}+2 \Phi^{4} B_{n l}=E_{n l} \Phi^{2} A_{n l} .
\end{gathered}
$$

The asymptotic expressions for the outer amplitudes in the boundary region, Eqs. (48)-(51), indicate that a simple expansion of the inner functions in powers of $\delta$ is insufficient. In order to ensure a match in the vicinity of the boundary layer to order $O(\epsilon)=O\left(\delta^{3}\right)$, the inner solutions must also include the non-trivial terms $\delta^{3} \ln \delta$ and $\delta^{3} \ln ^{2} \delta$. The eigenfunctions and eigenvalues are therefore expanded in the form:

$$
\begin{aligned}
A_{n l}(X) & =A_{n l}^{0}(X)+\delta A_{n l}^{1}(X)+\delta^{2} A_{n l}^{2}(X)+\delta^{3} A_{n l}^{3}(X)+\delta^{3} \ln \delta A_{n l}^{4}(X)+\delta^{3} \ln ^{2} \delta A_{n l}^{5}(X), \\
B_{n l}(X) & =B_{n l}^{0}(X)+\delta B_{n l}^{1}(X)+\delta^{2} B_{n l}^{2}(X)+\delta^{3} B_{n l}^{3}(X)+\delta^{3} \ln \delta B_{n l}^{4}(X)+\delta^{3} \ln ^{2} \delta B_{n l}^{5}(X), \\
E_{n l} & =E_{n l}^{0}+\delta E_{n l}^{1}+\delta^{2} E_{n l}^{2}+\delta^{3} E_{n l}^{3}+\delta^{3} \ln \delta E_{n l}^{4}+\delta^{3} \ln ^{2} \delta E_{n l}^{5} .
\end{aligned}
$$

Inserting these expansions into Eqs. (54) and (55), and taking into account the inner expansion for the condensate $\Phi(X)=\Phi_{0}(X)+\delta \Phi_{1}(X)+\cdots$, one obtains equations for the lowest-order inner functions:

$$
\begin{aligned}
& O\left(\delta^{0}\right):-\left(\Phi_{0}^{2} A_{n l}^{0}{ }^{\prime}\right)^{\prime}=0 \\
&-\left(\Phi_{0}^{2} B_{n l}^{0 \prime}\right)^{\prime}+2 \Phi_{0}^{4} B_{n l}^{0}=E_{n l}^{0} \Phi_{0}^{2} A_{n l}^{0} ; \\
& O\left(\delta^{1}\right): \quad-\left(\Phi_{0}^{2} A_{n l}^{1}{ }^{\prime}\right)^{\prime}-2\left(\Phi_{0} \Phi_{1} A_{n l}^{0 \prime}\right)^{\prime}-2 \Phi_{0}^{2} A_{n l}^{0{ }^{\prime}}=2 E_{n l}^{0} \Phi_{0}^{2} B_{n l}^{0} ; \\
&-\left(\Phi_{0}^{2} B_{n l}^{1}{ }^{\prime}\right)^{\prime}-2\left(\Phi_{0} \Phi_{1} B_{n l}^{0}{ }^{\prime}\right)^{\prime}-2 \Phi_{0}^{2} B_{n l}^{0{ }^{\prime}}+2 \Phi_{0}^{4} B_{n l}^{1}+8 \Phi_{0}^{3} \Phi_{1} B_{n l}^{0} \\
&=E_{n l}^{0}\left(\Phi_{0}^{2} A_{n l}^{1}+2 \Phi_{0} \Phi_{1} A_{n l}^{0}\right)+E_{n l}^{1} \Phi_{0}^{2} A_{n l}^{0},
\end{aligned}
$$

where a prime denotes a derivative with respect to $X$.

Equation (59) can be solved by taking $A_{n l}^{0}$ as a constant, and comparison with the first term of Eq. (48) shows that $A_{n l}^{0}=\rho_{n l}^{0}(1)$. In contrast, Eq. (60) is inhomogeneous, with the explicit solution

$$
B_{n l}^{0}(X)=-\frac{E_{n l}^{0} \rho_{n l}^{0}(1)}{\Phi_{0}(X)} \frac{d \Phi_{0}(X)}{d X}=-\frac{1}{2} E_{n l}^{0} \rho_{n l}^{0}(1) \frac{d \ln \Phi_{0}(X)^{2}}{d X} .
$$

By inserting the asymptotic expression (18) for $\Phi_{0}(X)$, it is simple to verify that $B_{n l}^{0}(X)$ matches the leading term of Eq. (49). 
The solution to Eq. (61) is readily found to be $A_{n l}^{1}(X)=\rho_{n l}^{0}{ }^{\prime}(1) X$, which is identical to the second correction term of the outer solution. The other correction $B_{n l}^{1}(X)$ satisfies a somewhat more complicated inhomogeneous equation (62). While a closed-form solution cannot be found, the behavior in the overlap region $X \rightarrow-\infty$ is easily obtained:

$$
B_{n l}^{1}(X) \sim-\frac{E_{n l}^{0} \rho_{n l}(1)}{2}\left(E_{n l}^{02}-\frac{1}{2}\right)-\frac{E_{n l}^{1} \rho_{n l}(1)}{2 X} .
$$

Matching the inner solution $B_{n l}^{1}$ with the outer function $\tilde{G}_{n l}^{0}$ to order $\delta$ requires $E_{n l}^{1}=0$. It then becomes possible to write an expression for $B_{n l}^{1}(X)$ valid for all $X$ :

$$
B_{n l}^{1}(X)=\frac{E_{n l}^{0} \rho_{n l}^{0}(1) \Xi_{a}(X)+E_{n l}^{0} \rho_{n l}^{0}{ }^{\prime}(1) \Xi_{b}(X)}{\Phi_{0}(X)},
$$

where the two functions $\Xi_{a}$ and $\Xi_{b}$ satisfy inhomogeneous equations similar to Eq. (17) for $\Phi_{1}$ :

$$
\begin{gathered}
-\Xi_{a}^{\prime \prime}+\left(X+3 \Phi_{0}^{2}\right) \Xi_{a}=4 \Phi_{0} \Phi_{0}^{\prime} \Phi_{1}-2 \Phi_{0} \frac{d}{d X}\left(X+\frac{\Phi_{1}}{\Phi_{0}}\right) \frac{d}{d X}\left(\frac{\Phi_{0}^{\prime}}{\Phi_{0}}\right), \\
-\Xi_{b}^{\prime \prime}+\left(X+3 \Phi_{0}^{2}\right) \Xi_{b}=X \Phi_{0} .
\end{gathered}
$$

For large negative $X$, the solutions approach $\Xi_{a} \sim \frac{1}{4}(-X)^{1 / 2}$ and $\Xi_{b} \sim-\frac{1}{2}(-X)^{1 / 2}$, so that $B_{n l}^{1}$ indeed matches the first correction term in Eq. (49). Each additional term in $\delta$ may be analyzed in a similar fashion; the procedure is straightforward but extremely tedious, so explicit solutions for the inner functions in the overlap region (which are lengthy) are omitted for brevity. It is important to note, however, that both inner functions $A_{n l}$ and $B_{n l}$ be properly matched to their outer region counterparts at each stage, in order to yield conditions on both the unknown coefficients and the eigenvalue corrections.

By following the above prescription in turn for each term in the inner expansion, it can be shown that all corrections to the eigenvalue with prefactors smaller than $O\left(\delta^{3}\right)$ (including the $O\left(\delta^{3} \ln \delta\right)$ and $O\left(\delta^{3} \ln ^{2} \delta\right)$ terms) must vanish identically. This result may be formally understood as follows. The density perturbation and the velocity-potential perturbation each have corrections to all orders both in the inner and outer regions; in contrast, the only correction term in the outer perturbation expansion of the energy (42) is of order $\delta^{3}$. In order to ensure a smooth asymptotic match between the bulk and surface amplitudes to each successive order, all the energy corrections appearing in the inner expansion (58) with coefficients smaller than $\delta^{3}$ must match to zero (the order $\delta$ case was considered explicitly above). The introduction of logarithmic terms to the outer perturbation expansions would give rise to additional contributions in the overlap region, leading to inconsistencies in the asymptotic match. The eigenvalue correction of order $\delta^{3}$ is finite, however: $E_{n l}^{3}=2 E_{n l}^{\epsilon}$. Thus,

$$
E_{n l}=E_{n l}^{0}+\frac{E_{n l}^{\epsilon}}{R^{4}}
$$

Eqs. (31) and (68) together yield the number-dependence of the excitation frequencies in the TF limit. The asymptotic match reveals an energy correction of order $\epsilon=R^{-4}$ exists, but unfortunately it does not indicate its magnitude nor its variation with $n, l$.

The present boundary-layer theory indicates that the energy correction of order $\delta^{3} \ln \delta$ vanishes identically. In contrast, a sum-rule approach 16.29 does yield a logarithmic correction to the eigenvalues, proportional to the ratio of the average kinetic energy (34) and external potential energy (32). This latter approach assumes that a single frequency exhausts the sum-rule. Such an assumption is thought to be valid in the hydrodynamic regime where a given perturbation excites essentially all of the atoms into a particular low-energy collective mode [30.31]. In the vicinity of the boundary layer, however, the density of atoms decreases considerably, and the single-mode approximation may become insufficient. In practice, any logarithmic correction to the energy eigenvalues would be experimentally or numerically detectable only if the magnitude of $E_{n l}^{\epsilon}$ were strongly dependent upon $n$ and $l$, or if the number of atoms were very low (small $R$ ). One may estimate the difference between $n=0$ energies obtained with and without a logarithmic correction, by assuming $E_{0 l}^{\epsilon} \approx l(l-1) \beta_{l} / 2$ [16] where $\beta_{l}=\log R$ and $\beta_{l}=1$, respectively. The deviation between the two approximations is independent of angular momentum, is largest when $\eta_{0} \approx 30$, and is at most $2 \%$ of the mode frequency when $l=10$. At present, therefore, the data are consistent with either theory.

The $(n, l)$-dependence of the energy correction $E_{n l}^{\epsilon}$ in Eq. (68) can not be found by conventional perturbation theory. This situation arises because the explicit integrals, which eliminate the logarithmic divergences, contain the 
nominally unperturbed inner functions; these functions, in turn, are themselves solutions of differential equations that implicitly include the perturbing terms. Consequently, we have considered the readily derived variational expression

$$
E_{j}=\frac{\int d V\left[\frac{1}{2} \tilde{\Psi}^{2} \nabla \tilde{F}_{j}^{*} \cdot \nabla \tilde{F}_{j}+2 \tilde{\Psi}^{4} \tilde{G}_{j}^{*} \tilde{G}_{j}+\frac{1}{2} \epsilon \tilde{\Psi}^{2} \nabla \tilde{G}_{j}^{*} \cdot \nabla \tilde{G}_{j}\right]}{\int d V \tilde{\Psi}^{2}\left(\tilde{G}_{j}^{*} \tilde{F}_{j}+\tilde{F}_{j}^{*} \tilde{G}_{j}\right)}
$$

which is stationary for small variations about the exact solutions $\tilde{F}_{j}$ and $\tilde{G}_{j}$. As a trial function, we use the unperturbed outer solutions in Eqs. (46), and the corresponding two-term inner solutions given in Eqs. (56) and (57); taken together, these expressions constitute uniform unperturbed solutions throughout all space. If the various integrals are divided at a point $x_{0}=1+\delta X_{0}$, with $X_{0} \ll-1$ and $\delta\left|X_{0}\right| \ll 1$, the correction terms arising from the behavior of the outer solution near the TF boundary precisely cancel with those from the inner boundary-layer solutions, leaving dimensionless integrals of order $\ln \delta$ through $\delta^{3} \ln ^{2} \delta$. The asymptotic match requires that only the term proportional to $\delta^{3}$ remains finite, and a detailed evaluation shows that the integrals up to order $\delta^{2} \ln \delta$ indeed vanish. The explicit calculation to order $\delta^{3}$ is prohibitive, however, due to the profusion of relevant terms.

The boundary-layer solutions given above now suffice to determine the approximate density fluctuation amplitude $\rho_{n l} \propto \tilde{\Psi}^{2} \tilde{G}_{n l}$ to order $\delta$ throughout the whole physical region. Inside the condensate, away from the boundary $\left(0 \leq x \leq x_{0}<1\right)$, the outer solution is simply the zero-order polynomial $\rho_{n l}^{0}(x)$ found by Stringari [16]. The uniformly matching inner solution $\Phi(X)^{2} B_{n l}(X)$ in the interval $X_{0} \leq X<\infty$ (where $x_{0}=1+\delta X_{0}$, with $\delta\left|X_{0}\right| \ll 1$ and $\left.X_{0} \ll-1\right)$ reduces to

$$
\rho_{n l} \approx-\rho_{n l}^{0}(1) \frac{d \Phi_{0}(X)^{2}}{d X}+2 \delta \rho_{n l}^{0}{ }^{\prime}(1) \Phi_{0}(X) \Xi_{b}(X)+2 \delta \rho_{n l}^{0}(1)\left[\Phi_{0}(X) \Xi_{a}(X)-2 \Phi_{1}(X) \frac{d \Phi_{0}}{d X}\right] .
$$

It is not difficult to verify that the third term in Eq. (70) vanishes for $X \rightarrow \pm \infty$, although it is nonzero inside the boundary region. For large positive $X$, each term in Eq. (70) vanishes exponentially, so that the density fluctuations become negligible beyond the surface region.

Figure 2 shows the spatial variation of the hydrodynamic amplitudes in the inner (boundary-layer) region, including corrections of order $\delta$ and $\delta^{2}$. The velocity potential perturbation $\phi_{n l}(X) \propto A_{n l}(X)$ (dot-dashed line) to order $\delta$ or $\delta^{2}$ is a linear or quadratic function of the inner coordinate $X$ and therefore diverges at large positive $X$. In contrast, both the density fluctuation $\rho_{n l}(X) \propto \Phi^{2}(X) B_{n l}(X)$ from Eq. (70) (solid line) and the perturbation in the current density $j_{n l} \propto \Phi^{2}(X) A_{n l}^{\prime}(X)$ (short dashed line) vanish exponentially in the limit $X \rightarrow \infty$. In fact, such behavior of the density and current-density amplitudes for $X \rightarrow \infty$ holds to all orders in $\delta$ as a direct consequence of the condensate wave function's exponential decay. The results of the boundary-layer theory differ from those obtained within the TF approximation, where the density-fluctuation amplitudes are merely finite at the TF radius.

The inner solutions provide a more detailed view of the behavior of these amplitudes near the condensate surface. In particular, the velocity potential and density perturbation coincide only in the outer region $X \ll-1$, reflecting the fact that in the limit $\epsilon \rightarrow 0$ the zeroth-order amplitudes $\tilde{F}_{n l}^{0}$ and $\chi_{0}^{2} \tilde{G}_{n l}^{0}$ obey the same differential equation (43). Since these unperturbed outer amplitudes are polynomials of order $2 n+l$ in the variable $x=1+\delta X$, the inner functions must be expanded to at least order $\delta^{2 n+l}$ in order to ensure a perfect asymptotic match.

\section{DISCUSSION}

The zero-temperature Thomas-Fermi description of an interacting dilute Bose-Einstein gas confined in an isotropic harmonic trap has been extended to include contributions from the condensate surface. For the condensate wave function, we have generalized the boundary-layer formalism of Dalfovo et al. [21], obtaining analytic expressions for the expectation values of the trap and interaction energy that include the leading corrections due to the surface layer and to the bulk condensate wave function. The resulting total ground state energy, which includes all terms of order $R^{-4} \ln R$ and $R^{-4}$, has not been evaluated previously.

The Bogoliubov equations for the excited states are rewritten in hydrodynamic form and solved to incorporate the boundary layer to third order in the boundary-layer thickness $\delta \propto R^{-4 / 3}$. This analysis provides a uniform extension of the hydrodynamic normal modes found by Stringari [16] beyond the TF condensate throughout the classically forbidden region. The lowest-order correction to the excitation frequencies has the form $E_{n l}^{\epsilon} / R^{4}$ (namely, of order $\epsilon \equiv R^{-4}$ ). Although a detailed calculation of the coefficient $E_{n l}^{\epsilon}$ appears prohibitive, the shift in excitation frequencies due to finite-number effects should be relevant for current experiments even when $\eta_{0} \gg 1$; both the sum-rule approach [16] and numerical calculations [32] indicate that $E_{n l}^{\epsilon}$ increases dramatically with both $n$ and $l$. 


\section{ACKNOWLEDGMENTS}

The authors would like to thank S. Stringari for stimulating discussions. D. L. F. is indebted to Barry Schneider for valuable suggestions regarding the numerical calculations. This work has been partially supported by the National Science Foundation under Grant No. 94-21888 (A. L. F.) and by the Natural Sciences and Engineering Research Council of Canada and the Ontario Centre for Materials Research (D. L. F.). In addition, D. L. F. appreciates the gracious hospitality of the Department of Physics at Stanford University.

\section{APPENDIX A: CONSTANTS FOR THE CONDENSATE}

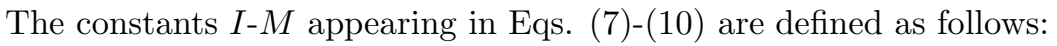

$$
\begin{aligned}
I & =\int_{-\infty}^{0} d X\left(\Phi_{0}^{2}+X\right)+\int_{0}^{\infty} d X \Phi_{0}^{2} \\
J & =\int_{-\infty}^{-1} d X\left(2 \Phi_{0}^{4}+\frac{5 X \Phi_{0}^{2}}{2}+\frac{X^{2}}{2}-\frac{3}{8 X}\right)+\int_{-1}^{\infty} d X\left(2 \Phi_{0}^{4}+\frac{5 X \Phi_{0}^{2}}{2}\right) \\
K & =\int_{-\infty}^{-1} d X\left(2 \Phi_{0}^{4}+\frac{7 X \Phi_{0}^{2}}{2}>+\frac{3 X^{2}}{2}-\frac{1}{8 X}\right)+\int_{-1}^{\infty} d X\left(2 \Phi_{0}^{4}+\frac{7 X \Phi_{0}^{2}}{2}\right) \\
L & =\int_{-\infty}^{-1} d X\left(\Phi_{0}^{4}-X^{2}-\frac{1}{2 X}\right)+\int_{-1}^{\infty} d X \Phi_{0}^{4} ; \\
M & =\int_{-\infty}^{-1} d X\left(\Phi_{0}^{4}+X \Phi_{0}^{2}-\frac{1}{4 X}\right)+\int_{-1}^{\infty} d X\left(\Phi_{0}^{4}+X \Phi_{0}^{2}\right) .
\end{aligned}
$$

Note that $K+L=J+M$. The integral $I$ can be evaluated analytically by setting the lower limit to $X_{0} \rightarrow-\infty$ and integrating by parts:

$$
I=\lim _{X_{0} \rightarrow-\infty} \frac{X_{0}^{2}}{2}-2 \int_{X_{0}}^{\infty} d X X \Phi_{0} \Phi_{0}^{\prime}
$$

Multiplying Eq. (16) by $\Phi_{0}^{\prime}$ and integrating, one readily obtains:

$$
\int_{X_{0}}^{\infty} d X X \Phi_{0} \Phi_{0}^{\prime}=\frac{1}{2}\left[\Phi_{0}^{\prime 2}-\frac{\Phi_{0}^{4}}{2}\right]_{X_{0}}^{\infty}
$$

The asymptotic behaviors $(18)$ and $\Phi_{0}(X \rightarrow \infty)=0$ immediately give the result $I=0$.

The derivation of the expressions for $J$ and $K$ makes use of the relation

$$
\begin{aligned}
\int_{-\infty}^{\infty} d X \Phi_{0} \Phi_{1}=- & \frac{23}{16}-\frac{3}{4} \int_{-\infty}^{-1} d X X^{2} \\
& +\int_{-\infty}^{\infty} d X\left(2 \Phi_{0}^{4}+\frac{3}{2} X \Phi_{0}^{2}\right)
\end{aligned}
$$

which may be verified by integrating by parts, comparing with Eqs. (16)-(19), and employing the readily proved identity

$$
\int_{-\infty}^{\infty} d X \Phi_{0}^{\prime 2}=\frac{1}{2}-\int_{-\infty}^{\infty} d X\left(\Phi_{0}^{4}+X \Phi_{0}^{2}\right)
$$

Furthermore, with the identity

$$
2 \int_{-\infty}^{\infty} d X X \Phi_{0}^{2}=-\frac{1}{6}-\int_{-\infty}^{-1} d X X^{2}-\int_{-\infty}^{\infty} d X \Phi_{0}^{4}
$$

which may be easily confirmed by integrating by parts and making use of the governing equation $(16)$ for $\Phi_{0}(X)$, the expressions (A2)- (A5) are found to be related: 


$$
\begin{aligned}
J & =\frac{3}{4} L-\frac{5}{24} \\
K & =\frac{1}{4} L-\frac{7}{24} \\
M & =\frac{1}{2} L-\frac{1}{12} .
\end{aligned}
$$

\section{APPENDIX B: CONSTANTS FOR THE EXCITATIONS}

The constants appearing in the asymptotic expansions of the outer amplitudes $F_{n l}^{0, \epsilon}$ and $G_{n l}^{0, \epsilon}$, Eqs. (48)-(51), are explicitly written (where $\rho_{0}(1) \equiv \rho_{n l}^{0}(1)$ and $\left.E_{0} \equiv E_{n l}^{0}\right)$ :

$$
\begin{aligned}
\rho_{0}^{\prime}(1) & =E_{0}^{2} \rho_{0}(1) ; \\
\rho_{0}^{\prime \prime}(1) & =\frac{1}{2}\left[E_{0}^{4}-3 E_{0}^{2}+l(l+1)\right] \rho_{0}(1) ; \\
\rho_{0}^{\prime \prime \prime}(1) & =\frac{1}{6}\left[E_{0}^{6}-10 E_{0}^{4}+5 E_{0}^{2}(l(l+1)+5)-13 l(l+1)\right] \rho_{0}(1) ; \\
G_{a}^{0} & =\rho_{0}(1) ; \\
G_{b}^{0} & =\left[E_{0}^{2}-\frac{1}{2}\right] \rho_{0}(1) ; \\
G_{c}^{0} & =\frac{1}{4}\left[E_{0}^{4}-5 E_{0}^{2}+l(l+1)+1\right] \rho_{0}(1) ; \\
G_{d}^{0} & =\frac{1}{72}\left[2 E_{0}^{6}-29 E_{0}^{4}+5 E_{0}^{2}(2 l(l+1)+19)-35 l(l+1)-9\right] \rho_{0}(1) ; \\
F_{a}^{\epsilon} & =0 ; \\
F_{b}^{\epsilon} & =0 ; \\
F_{c}^{\epsilon} & =\frac{1}{16}\left[E_{0}^{4}-7 E_{0}^{2}+3 l(l+1)\right] \rho_{0}(1) ; \\
F_{d}^{\epsilon} & =C_{a}^{\epsilon} ; \\
F_{e}^{\epsilon} & =C_{b}^{\epsilon} ; \\
F_{f}^{\epsilon} & =-\frac{1}{16}\left[E_{0}^{4}-E_{0}^{2}(4 l(l+1)+3)+5 l(l+1)\right] \rho_{0}(1) ; \\
G_{a}^{\epsilon} & =-\frac{3}{8} \rho_{0}(1) ; \\
G_{b}^{\epsilon} & =-\frac{1}{8}\left[E_{0}^{2}-12\right] \rho_{0}(1) ; \\
G_{c}^{\epsilon} & =-\frac{1}{32}\left[E_{0}^{4}-23 E_{0}^{2}-11 l(l+1)+66\right] \rho(1) ; \\
G_{d}^{\epsilon} & =-\frac{1}{32}\left[E_{0}^{6}-15 E_{0}^{4}-E_{0}^{2}(3 l(l+1)-70)+8 l(l+1)-66\right] \rho_{0}(1)+C_{a}^{\epsilon} ; \\
G_{e}^{\epsilon} & =C_{b}^{\epsilon} ; \\
G_{f}^{\epsilon} & =-\frac{1}{16}\left[E_{0}^{4}-E_{0}^{2}(4 l(l+1)+3)+5 l(l+1)\right] \rho_{0}(1),
\end{aligned}
$$

where $C_{a}^{\epsilon}$ and $C_{b}^{\epsilon}$ are constants of integration.

[1] M. H. Anderson, J. R. Ensher, M. R. Matthews, C. E. Wieman, and E. A. Cornell, Science 269, 198 (1995).

[2] K. B. Davis, M.-O. Mewes, M. R. Andrews, N. J. van Druten, D. S. Durfee, D. M. Kurn, and W. Ketterle, Phys. Rev. Lett. 75, 3969 (1995).

[3] C. C. Bradley, C. A. Sackett, and R. G. Hulet, Phys. Rev. Lett. 78, 985 (1997).

[4] N. N. Bogoliubov, J. Phys. (Moscow) 11, 23 (1947).

[5] E. P. Gross, Nuovo Cimento 20, 454 (1961).

[6] L. P. Pitaevskii, Zh. Eksp. Teor. Fiz. 40, 646 (1961) [Sov. Phys. JETP 13, 451 (1961)].

[7] M. Edwards and K. Burnett, Phys. Rev. A 51, 1382 (1995).

[8] P. A. Ruprecht, M. J. Holland, K. Burnett, and M. Edwards, Phys. Rev. A 51, 4704 (1995).

[9] M. Edwards, P. A. Ruprecht, K. Burnett, and C. W. Clark, Phys. Rev. Lett. 77, 1671 (1996).

[10] F. Dalfovo and S. Stringari, Phys. Rev. A 53, 2477 (1996).

[11] G. Baym and C. J. Pethick, Phys. Rev. Lett. 76, 6 (1996).

[12] A. L. Fetter, J. Low Temp. Phys. 106, 643 (1997). 
[13] A. L. Fetter, Phys. Rev. A 53, 4245 (1996).

[14] W.-C. Wu and A. Griffin, Phys. Rev. A 54, 4204 (1996).

[15] A. L. Fetter and D. Rokhsar, Phys. Rev. A 57, 1191 (1998).

[16] S. Stringari, Phys. Rev. Lett. 77, 2360 (1996).

[17] M. Edwards, R. J. Dodd, C. W. Clark, and K. Burnett, J. Res. NIST 101, 553 (1996).

[18] P. A. Ruprecht, M. Edwards, K. Burnett, and C. W. Clark, Phys. Rev. A 54, 4178 (1996).

[19] D. S. Jin, J. R. Ensher, M. R. Matthews, C. E. Wieman, and E. A. Cornell, Phys. Rev. Lett. 77, 420 (1996).

[20] M.-O. Mewes, M. R. Andrews, N. J. van Druten, D. M. Kurn, D. S. Durfee, C. G. Townsend, and W. Ketterle, Phys. Rev. Lett. 77, 988 (1996).

[21] F. Dalfovo, L. P. Pitaevskii, and S. Stringari, Phys. Rev. A 54, 4213 (1996).

[22] A. L. Fetter, Ann. Phys. (N.Y.) 70, 67 (1972).

[23] See, for example, C. M. Bender and S. A. Orszag, Advanced Mathematical Methods for Scientists and Engineers (McGrawHill, NY, 1978), Chap. 9.

[24] E. Lundh, C. J. Pethick, and H. Smith, Phys. Rev. A 55, 2126 (1996).

[25] M. I. Ablowitz and H. Segur, Phys. Rev. Lett. 38, 1103 (1977).

[26] A. Gonzalez and A. Perez, cond-mat/9802023, have used Padé approximants to interpolate between the TF limit and the nearly ideal gas, in both three and two dimensions (note that their definition of $R$ differs from ours).

[27] S. Stringari, private communication.

[28] See, for example, Handbook of Mathematical Functions with Formulas, Graphs, and Mathematical Tables, edited by M. Abramowitz and I. A. Stegun (Nat. Bur. Stds., Washington, DC, 1968), Chap. 15.

[29] F. Zambelli and S. Stringari, cond-mat/9805068.

[30] R. D. Puff, Phys. Rev. 65, A406 (1965).

[31] S. Stringari, Phys. Rev. B 46, 2974 (1992).

[32] B. I. Schneider and D. L. Feder, unpublished.

FIG. 1. The contribution of the kinetic energy in units of $\hbar \omega_{0}$ is shown as a function of the universal scaling parameter $\eta_{0}=N_{0} a / d_{0}$. The kinetic energy per particle is calculated numerically by direct integration of the GP equation (solid bold) and analytically by the boundary-layer theory (dashed bold), whose result is given in Eq. (34). The dashed thin line is the difference between the exact total energy per particle (computed numerically) and the full boundary-layer theory in Eq. (35). The solid thin line corresponds to the similar difference keeping only the approximate result of the TF theory [the first term of Eq. (35)]. The total energies per particle for a restricted range of $\eta_{0}$ are shown in the inset for the exact (solid), boundary-layer (dashed), and TF (long dashed) theories. On this restricted scale, the curves for the boundary-layer and exact results coincide.

FIG. 2. The (unnormalized) hydrodynamic amplitudes and condensate wave function in the boundary layer are shown as a function of inner coordinate $X$. Bold and thin lines correspond to results calculated numerically to order $\delta$ and $\delta^{2}$, respectively. The universal parameter is $\eta_{0}=1000$ giving $\delta \approx 0.06$, and therefore $X=-8$ corresponds to $x \approx 0.5$. With units chosen appropriately, the velocity potential to order $\delta$ (bold dot-dashed line) and the density perturbation [bold solid line to order $\delta$, from Eq. (70)] coincide in the overlap region $X \ll-1$; while the former diverges as $X \rightarrow \infty$, the latter decays exponentially. The perturbations in the current density (dashed lines) to order $\delta$ (bold) and $\delta^{2}$ (thin) are respectively linear and quadratic in the overlap region and decay exponentially at large distances. The results are presented for $(n, l)=(0,2)$; the overall sign of the inner hydrodynamic amplitudes is odd in $n$, and the magnitude of the asymptotic slope (for large negative $X$ ) increases with $l$. The inset shows the various amplitudes in the bulk region as a function of outer coordinate $x$. In the outer region, the velocity potential and density perturbation coincide (shown as the solid line); the former matches smoothly with that from the inner region to order $\delta^{2}$ (thin dot-dashed line). A perfect asymptotic match of the outer current density $\propto x\left(1-x^{2}\right)($ dashed line) to its inner counterpart would require an inner expansion to order $\delta^{3}$. 


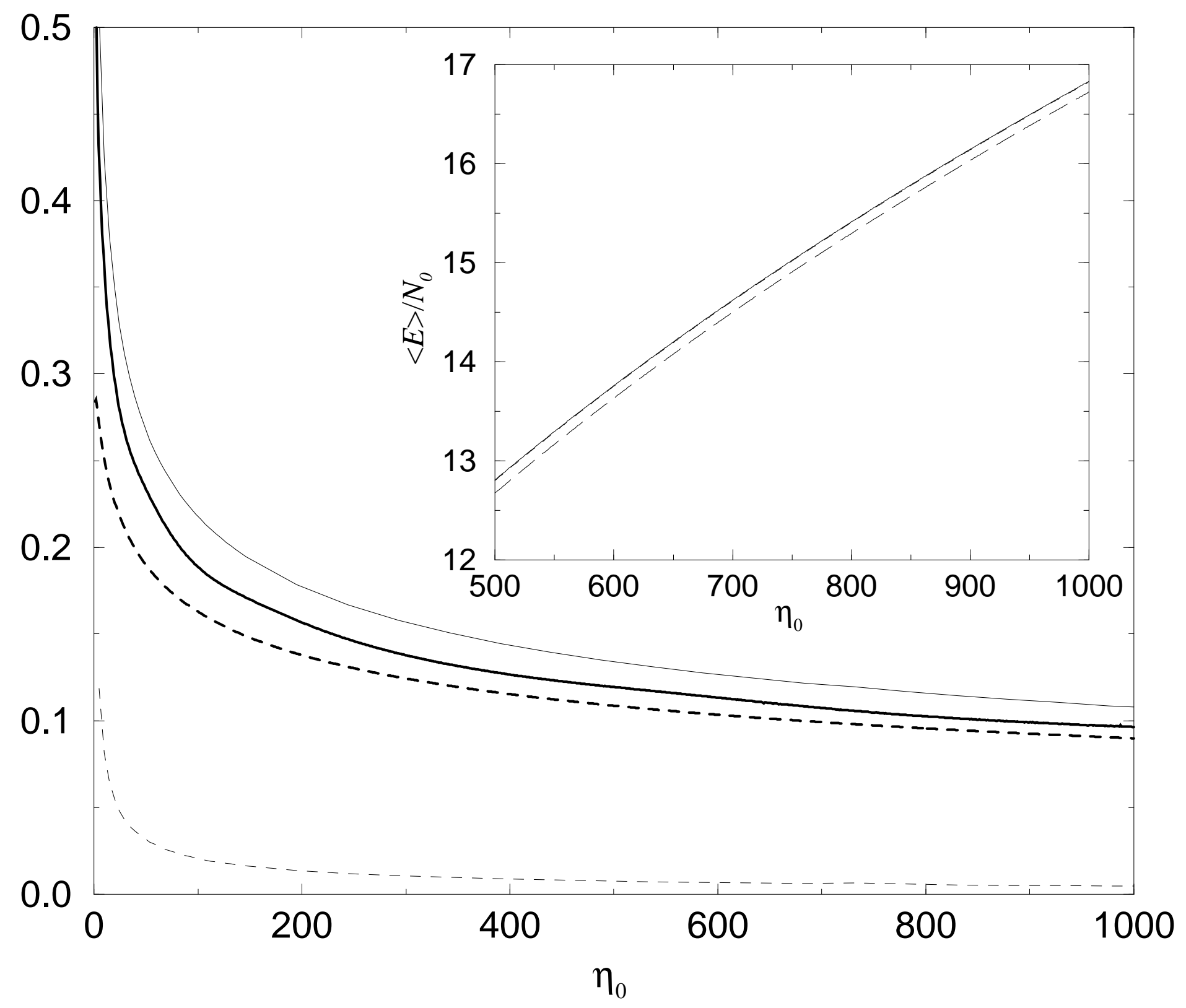




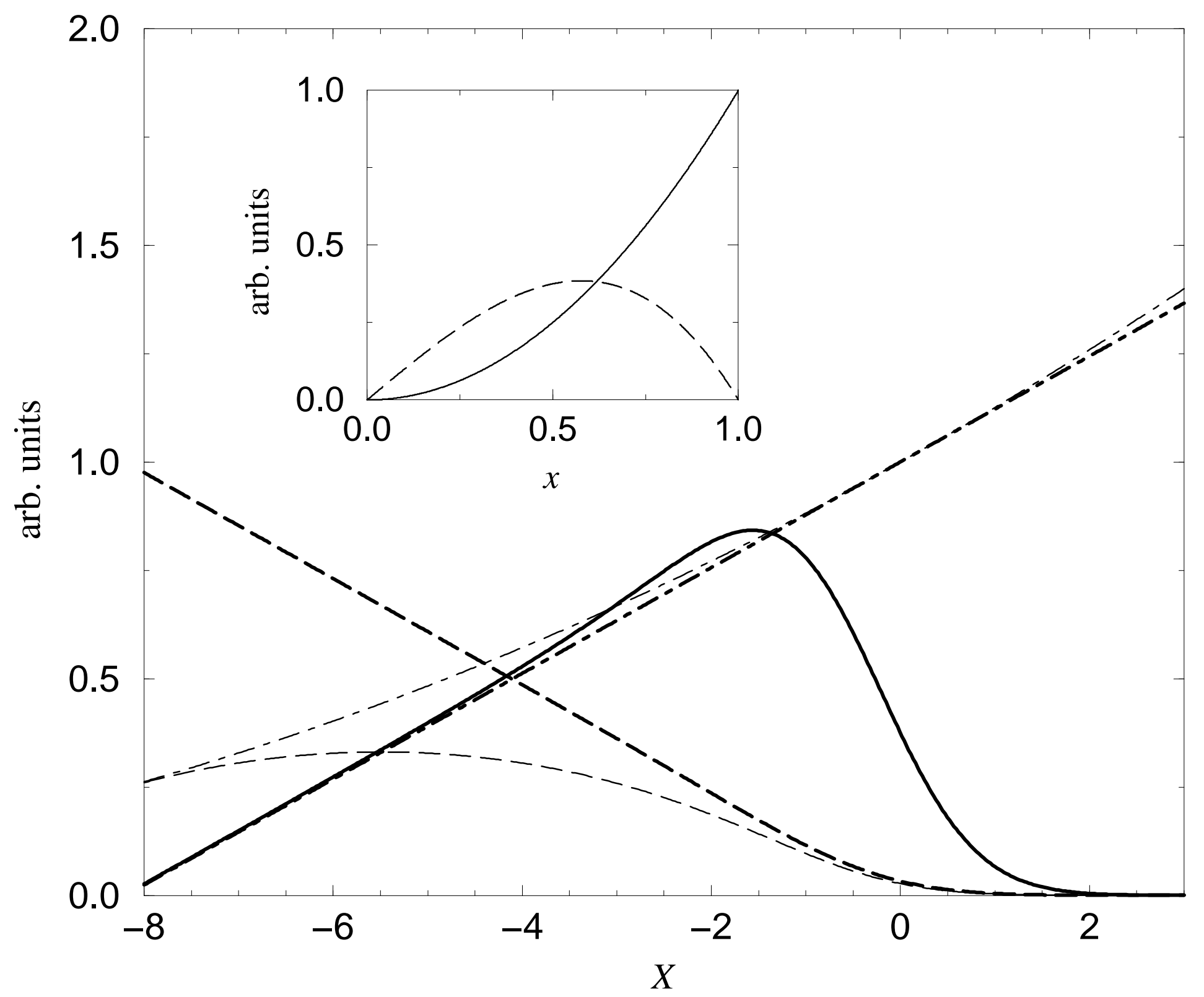

